\title{
Natural Interactive System for Hemispatial Neglect Rehabilitation
}

\author{
Gianpaolo D'Amico, Lea Landucci, and Daniele Pezzatini \\ MICC - Media Integration and Communication Center, \\ University of Florence, Italy \\ http://www.micc.unifi.it
}

\begin{abstract}
Experimentation of natural interaction principles and advanced interactive solutions for medical treatment of the neglect syndrome. Novel tools are provided for improving rehabilitation and evaluation of patients and overcome the conventional pen-and-paper approach. This project is the result of a multidisciplinary collaboration among Media Integration and Communication Center, the Faculty of Psychology of the University of Florence and Montedomini A.S.P., a public agency for disabled elders and healthcare services in Italy.
\end{abstract}

Keywords: User Interfaces, Information Interfaces and Presentation, Life and Medical Sciences.

\section{Introduction}

Hemispatial neglect (or neglect syndrome) is a neuropsychological condition in which, after a damage to one hemisphere of the brain, a deficit in attention to and awareness of one side of space is observed. It is defined by the inability of a person to process and perceive stimuli on one side of the body or environment that is not due to a lack of sensation. [1] For example a stroke affecting the right lobe of the brain can lead to neglect for the left side of the field of view, causing a patient to behave as if such side of sensory space does not exist. In such conditions the patient could be no more able to pass a door without hitting the jamb, or to eat the food on a dish without leaving the left half part completely untouched. Unfortunately, the only treatment consists of finding ways to bring the patient's attention toward the left, usually done incrementally, by going just a few degrees past midline, and progressing from there. Moreover the difficulty degree of such task increases with the age of the patient.

The Behavioural Inattention Test (BIT) 2] is a 15-item standardized test battery for assessing visual neglect, consisting of 6 of the most commonly used pen-and-paper tests (line crossing, letter cancellation, star cancellation, figure copying, line bisection, and free drawing) and 9 behavioral tasks (picture scanning, telephone dialing, menu reading, article reading, telling and setting the time, coin sorting, address and sentence copying, map navigation, and card sorting) (see fig (1). One component of the test battery is a simple test described by Albert [3] in which patients cross out lines on a sheet of paper (see fig. 1 - b);

A. Petrosino, L. Maddalena, P. Pala (Eds.): ICIAP 2013 Workshops, LNCS 8158, pp. 501-508, 2013.

(C) Springer-Verlag Berlin Heidelberg 2013 


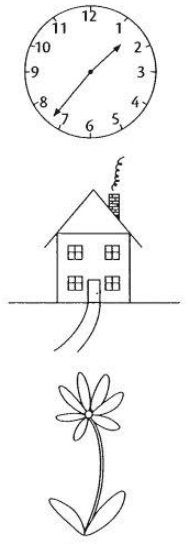

(a)

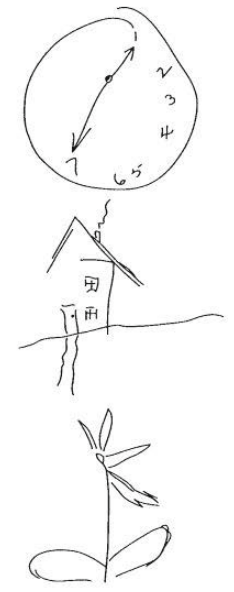

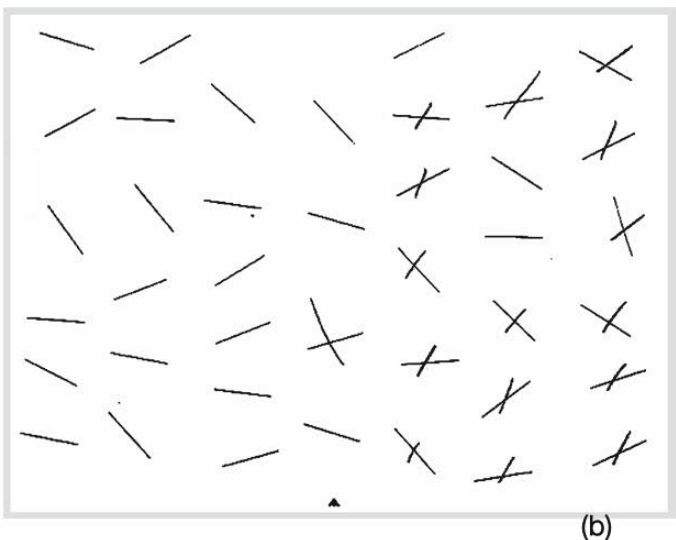

Fig. 1. Set of pen-and-paper tests from the BIT battery: (a) figure copying and (b) Albert's test

this is easy to administer and related closely to neglect diagnosed by the test battery as a whole [4]: that is the reason why we focused on Albert's test.

As reported by the medical staff we interviewed, the main issue in Albert's test is that particular negative results can be due to lack of stimulation, especially for elderly patients. That is why the idea of this work is to translate analog Albert's tests in digital tests able to stimulate the patient's attention.

For such delicate process we have exploited the help of the department of psychology at the University of Florence that suggested some real-world contexts able to put patients at ease and encourage them to actively participate in all the test and training phases.

\section{Related Work}

There is a wide variety of solutions for the study of the neglect syndrome still using paper based tools, as in [11, where drawing-based pencil-and-paper tests enable objectivity, repeatability and diagnostic capability in the scoring process of the disease. Anyway there is another part of research which experiments solutions for the development of neuropsychological assessment and rehabilitation tools via the adoption of computer-based advanced technologies.

A former approach is based on Virtual reality (VR), which is showed to be effective for dealing with cognitive and functional impairments due not only to traumatic brain injury, but also to neurological disorders and learning disabilities [6] 10]. VR-DiSTRO[5] is a solution which uses shutter stereoscopic glasses, a force feedback interface and a software, in order to provide an immersive experience and then estimate its accuracy and usability for patients affected by acute neglect syndrome. A similar approach is presented in [8], where a 3Dhaptic virtual world is seen through stereo shutter-glasses, where hemispatial 
neglect patients interact by manipulating a haptic interface. A different approach is discussed in [7, where post-stroke neglect in the extrapersonal space was evaluated. Authors modeled a street crossing in VR, in which a battery of thirtytwo patients with right-hemispheric stroke were asked to control a virtual avatar safe from a traffic accident. In [14 authors studied unilateral spatial neglect following stroke in recovered post-stroke patients with residual symptoms of unilateral spatial neglect. They compared the assessment and treatment results from standard paper and pencil tests with their VR solution. SeeMe is a system in which participants see themself in real time in a projected screen while they assess functional tasks touching virtual balls. A single screen-mounted camera and vision-based tracking system capture and convert the user's movements for processing on the big display.

Other computer-based approaches which differ from VR technology are presented in [12, where a game-based solution composed of a webcam, a computer and a video projector is used to guide the patient in exploring the space (included the neglected hemispace) with the goal to reach for targets with different levels of difficulty. In [13] a system to measure eye and head-neck movement is presented for the evaluation of the unilateral spatial neglect fields. Eye motion was estimated via a small CMOS camera attached to a head unit, while the head-neck movement was processed via a high-vision video camera attached to the front of the patient. In [9] authors proposed a computerized version of the cancellation test via the adoption of a touchscreen interface to large groups of control subjects and unilaterally brain-damaged patients (unilateral and nonunilateral spatial neglect).

\section{The System}

The proposed solution is represented by an interactive environment in which patients are asked to perform different tasks in order to estimate their neurocognitive condition (testing phase) and to support rehabilitation activities (training phase). Tasks, that have been defined under the supervision of a team of psychologists, consist of predefined exercises to address the following elements: memory, attention, perceptual disturbances, visualspatial disturbances and difficulties in executive functions. The main idea is to allow patients to interact with digital content through gestures in order to perform tasks similar to those that would occur in their daily life. On the other hand, we wanted to provide medical staff with an advanced tool to customize tasks configurations and to analyze data obtained from patients activity.

The system is composed by two main parts: a touch based interactive surface for patients and a desktop based application for medical staff. The interactive surface is visualized on a wide LCD screen (52") with multitouch capabilities provided by an infrared based overlay. The screen is mounted on a reclining and height-adjustable table, so that every patient could easily interact with every part of the screen. The chosen interface metaphor for the proposed use case is 
the action of cleaning a dirty table (see fig. 2). Patients are asked to move a real sponge on the interactive table in order to physically erase virtual dust or spots displayed on the screen.

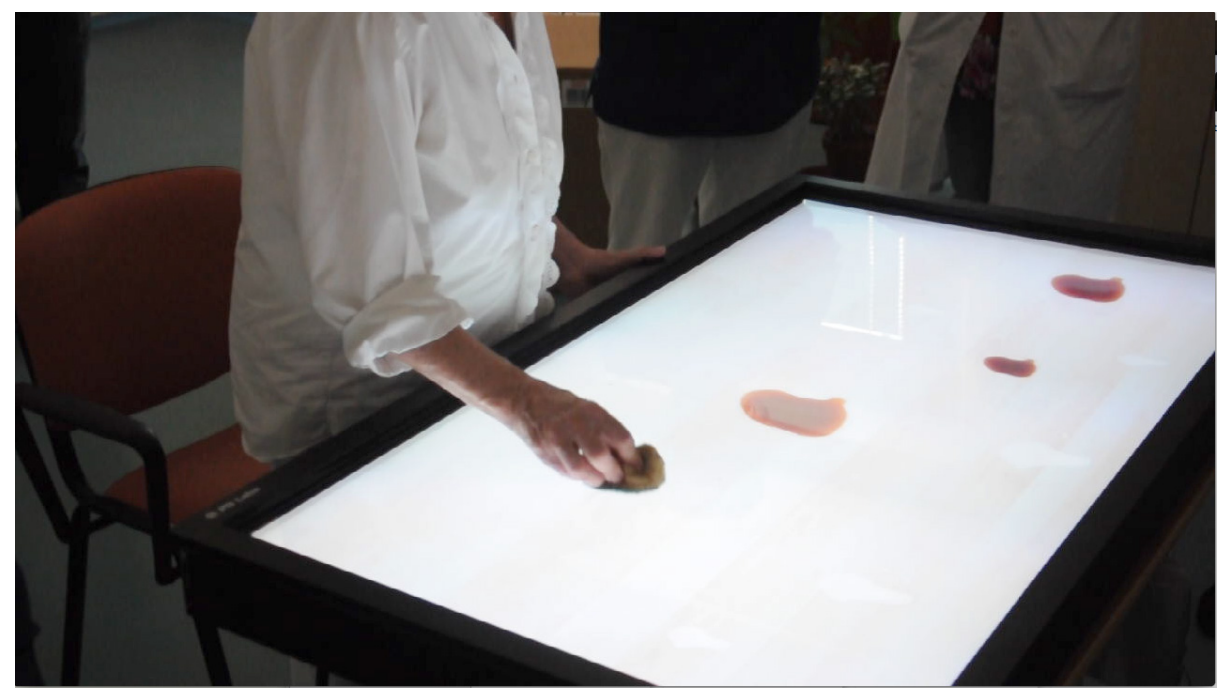

Fig. 2. A patient performing the task of cleaning the interactive table from coffee spots

The testing phase consists of collecting information on the severity of the condition and progress of the patient, whilst the training phase encourages the exploration of the neglected hemifield through various procedures. The set of the spots (visual stimuli) displayed on the table can be configured according to four parameters: number, location, size and nature (e.g. coffee, oil, water; see fig 3).

Doctors and therapists can create different configurations of tasks using a desktop based GUI in order to tune difficulty according to patient conditions. As an example, a training task could be configured with a particular spatial distribution of stimuli on the basis of patients neglected hemifield. Therapists can also add audiovisual feedback to stimuli (e.g. blinking light, sounds etc.) that can be triggered during the training session helping patients to complete the task.

All the activities during the rehabilitation sessions are tracked and recorded in a database so that a personal history of each patient can be evaluated in order to estimate performances in terms of accuracy (number of erased spots), time spent in accomplishing the task and trajectory of movements. In this way, medical staff can work with a novel diagnostic tool which provides useful information, statistics, charts and high-level data for the evaluation of patients (see fig 4).

Both the desktop application and the interactive rehabilitation interface are developed using the AIR (Adobe Integrated Runtime) framework, allowing to bring ActionScript and Flex code into a native desktop installer. The multitouch 


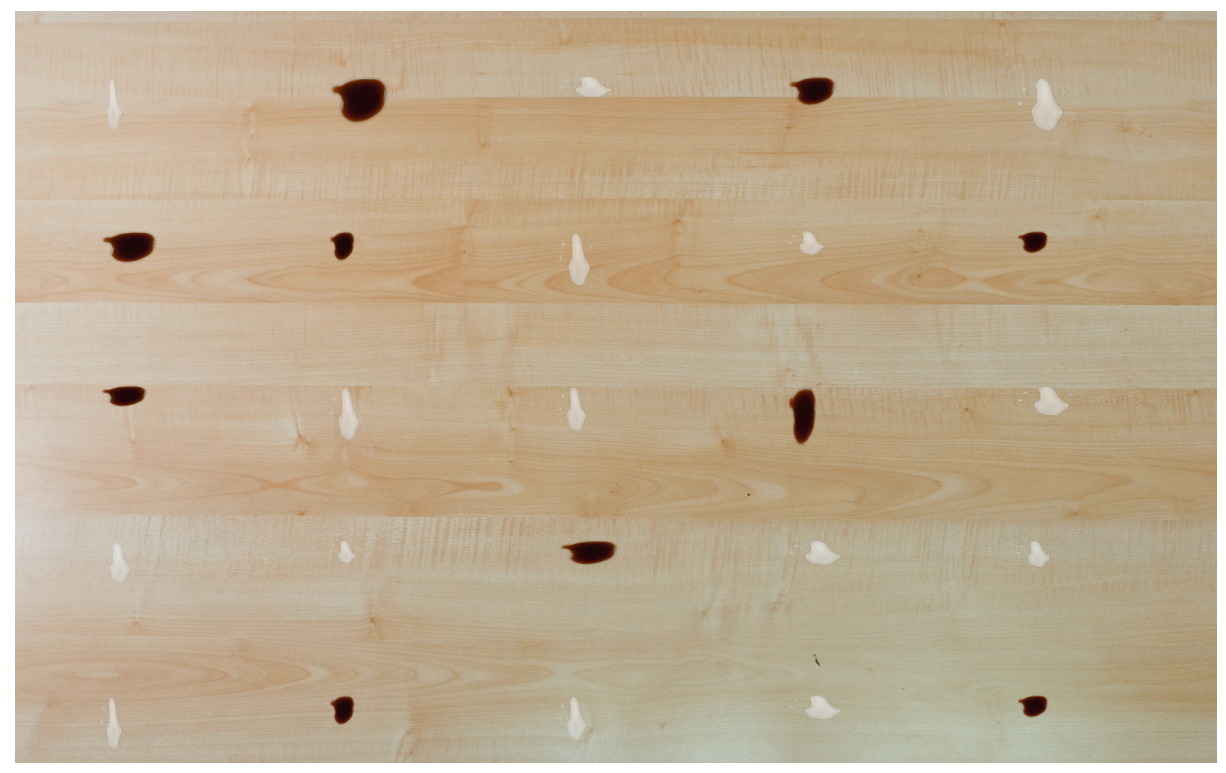

Fig. 3. Interactive table with water and coffee spots
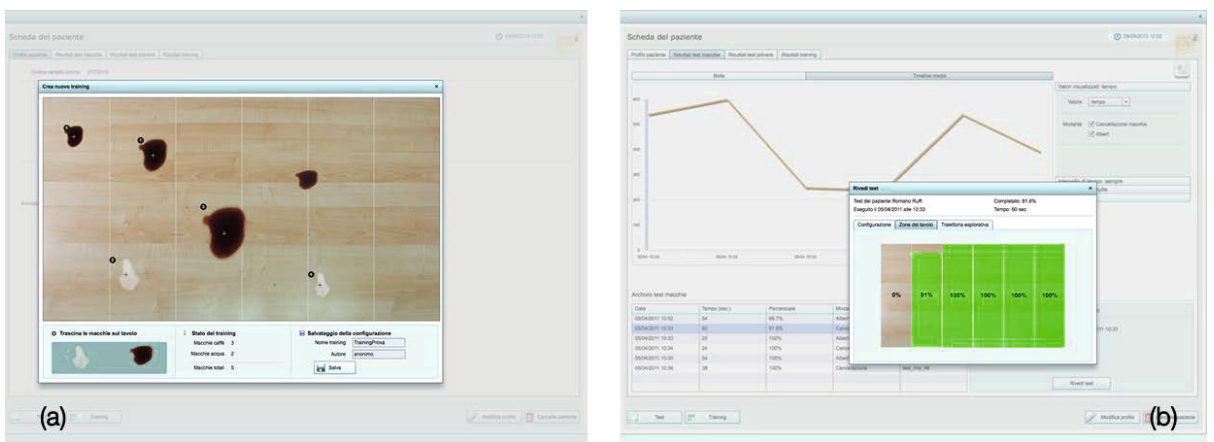

Fig. 4. (a) drag and drop GUI for training configuration and (b) statistical analysis tools 
overlay sends data on tracked fingers and objects to the main application using the TUIO protocol [15].

\section{Experimental Results}

The real-world testing phase occurred thanks to the collaboration with Montedomini A.S.P. who exploited the system for the experimental rehabilitation of approximately 30 patients in the last two years.

Taking into account a sample of ten representing patients, for whom we had available the traditional test of Albert as a check-in test for the experimentation, we discovered not only that the digital training phase has produced superior results than standard training, but also that the system has also led to a partial psycho-motor rehabilitation.

As expected, a table sprinkled with (digital) spots or dust has a strong affordance so that the instinct to clean is greater than that of concluding a penand-paper test: as shown in fig [5, in the majority of cases the patients had lower performance on the standard Albert's test compared to the interactive digital test, even before the training phase.

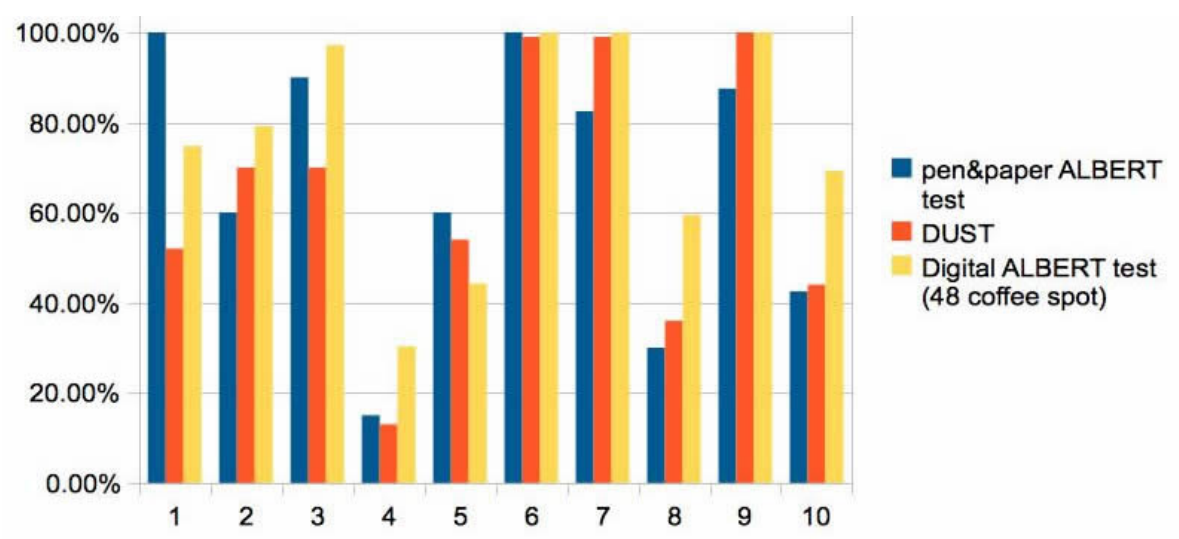

Fig. 5. Patients results for check-in test

The patients were then treated with a training phase of variable duration (one to three months depending on the patients) using the visual and auditory interface facilities.

The check-out tests performed after training phase had great results: as shown in fig 6 , the patients improved the awareness of the left side of view (this was proved not only with the digital test but also with the traditional one).

The physical therapists who have followed them have also recorded an improvement in their physical condition. In fact, in order to reach the entire surface of the table, patients have unconsciously also worked on their body: the lower 
part has worked to support the whole body (training of body-balance) while the upper part had a benefit due to the stretching of arms performed in order to reach the upper and side areas of the table (such effect has obviously occurred also in patients forced to use the table on wheelchairs).

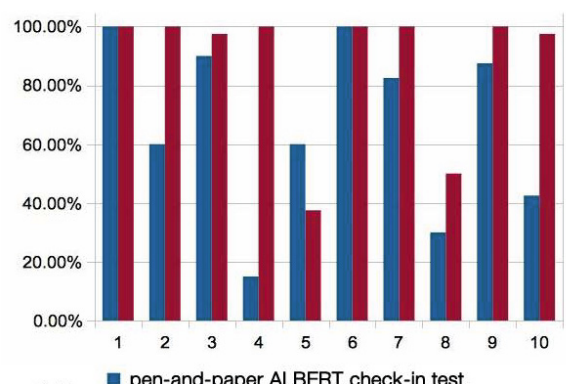

(a)

- pen-and-paper ALBERT check-in test

- pen-and-paper ALBERT check-out test

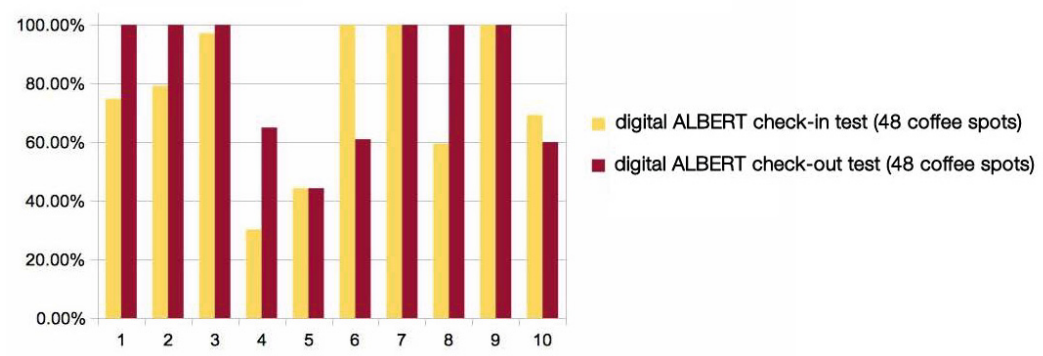

(c)

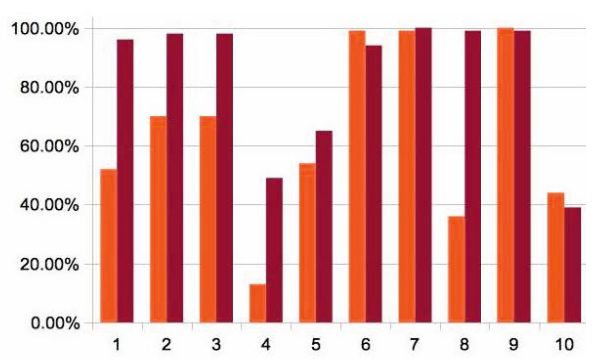

(b) DUST check-in test (b) DUST check-out test

Fig. 6. Patients results for check-out test

Finally, medical staff has recognized added value in the efficacy of the performance analysis process due to the use of the computerised analysis tools provided by the system.

\section{Conclusions and Future Work}

The developed system allows effective analysis of the clinical status of patients affected by the neglect syndrome and a fine tool for the rehabilitation training. The idea is to use a real-world context in order to stimulate elder patients to perform tests and to improve their clinical condition through the training phase. First experimental results are very positive and encourage us to continue on this path. Future work includes the improvement of the evaluation on patients and the development of new natural interactive contexts for the rehabilitation also exploiting dynamic scenarios. 


\section{References}

1. Unsworth, C.A.: Cognitive and Perceptual Dysfunction. In: Physical Rehabilitation, pp. 1149-1185 (2007)

2. Wilson, B.A., Cockburn, J., Halligan, P.W.: Behavioural Inattention Test. Thames Valley Test Company Ltd., Titchfield (1987)

3. Albert, M.: A simple test of visual neglect. Neurology 23(6), 58-64 (1973)

4. Fullerton, K.J., Mcsherry, D., Stout, R.W.: Albert's test: a neglected test of perceptual neglect. The Lancet 327(8478) (February 22, 1986)

5. Fordell, H., Bodin, K., Bucht, G., Malm, J.: A virtual reality test battery for assessment and screening of spatial neglect. Acta Neurol Scand. 123, 167-174 (2011)

6. Rizzo, A.A., Schultheis, M., Kerns, K.A., Mateer, C.: Analysis of assets for virtual reality applications in neuropsychology. Neuropsychological Rehabilitation 14(1/2), 207-239 (2004)

7. Kim, D.Y., Ku, J., Chang, W.H., Park, T.H., Lim, J.Y., Han, K., Kim, I.Y., Kim, S.: Assessment of post-stroke extrapersonal neglect using a three-dimensional immersive virtual street crossing. Acta Neurol Scand. 121, 171-177 (2010)

8. Baheux, K., Yoshikawa, M., Tanaka, A., Seki, K., Handa, Y.: Diagnosis and Rehabilitation of Patients with Hemispatial Neglect Using Virtual Reality. In: Proceedings of the 26th Annual International Conference of the IEEE EMB, San Francisco, CA, USA (2004)

9. Rabuffetti, M., Farina, E., Alberoni, M., Pellegatta, D., Appollonio, I., Affanni, P., Forni, M., Ferrarin, M.: Spatio-Temporal Features of Visual Exploration in Unilaterally Brain-Damaged Subjects with or without Neglect: Results from a Touchscreen Test. PLoS One 7(2) (2012)

10. Rose, F.D., Brooks, B.M., Rizzo, A.A.: Virtual Reality in Brain Damage Rehabilitation: Review. Cyberpsychol. Behav. 8(3), 241-262 (2005)

11. Liang, Y., Fairhurst, M.C., Guest, R.M., Potter, J.M.: A Learning Model for the Automated Assessment of Hand-Drawn Images for Visuo-Spatial Neglect Rehabilitation. IEEE Trans. Neural Syst. Rehabil. Eng. 18(5), 60-70 (2010)

12. Borghese, A.N., Sedda, A., Mainetti, R., Ronchetti, M., Pasotti, F., Bottini, G.: A reliable low-cost platform for neglect Virtual Rehabilitation. In: International Conference on Virtual Rehabilitation, Switzerland, June 27-29 (2011)

13. Sugihara, S., Miyasaka, T., Tanaka, T.: A study of assessment of unilateral spatial neglect using a system for motion analysis of eyes and a head-neck. In: IEEE/SICE International Symposium on System Integration, January 29, pp. 67-70 (2009)

14. Sugarman, H., Weisel-Eichler, A., Burstin, A., Brown, R.: Use of Novel Virtual Reality System for the Assessment and Treatment of Unilateral Spatial Neglect: A Feasibility Study. In: International Conference on Virtual Rehabilitation, Switzerland, June 27-29 (2011)

15. Kaltenbrunner, M., Bovermann, T., Bencina, R., Costanza, E.: TUIO - A Protocol for Table-Top Tangible User Interfaces. In: Proceedings of the 6th International Workshop on Gesture in Human-Computer Interaction and Simulation, Vannes, France (2005) 